\title{
Research on Influencing Factors of Knowledge Sharing in Supply Chain Enterprises under Blockchain Environment
}

\author{
Yi CHAl, Quanxi LI*
}

\begin{abstract}
There are many participating companies involved in the supply chain system. In order to achieve multi-enterprise, multi-resource-oriented service product information sharing, it is necessary to have the ability to match transactions in a highly credible environment. In the current supply chain system, the core enterprise generally builds a centralized supply chain resource-sharing platform to realize knowledge sharing, and the security of the system completely depends on the core enterprise. In addition, the cost of trust establishment is very high. It is urgent to establish a reasonable trust mechanism. On this basis, the factors affecting knowledge sharing and the problems to solve are analyzed, and the plan and factors of using blockchain technology to build a knowledge-sharing platform are proposed. Finally, the conceptual model of the blockchain-based knowledge-sharing platform is designed, and its business process and economic model are emphasized to provide ideas for breaking through the bottleneck of the supply chain system.
\end{abstract}

Keywords: blockchain; centralization; knowledge sharing; supply chain

\section{INTRODUCTION}

The knowledge sharing and dissemination among supply chain enterprises is conducive to improving the knowledge sharing of the entire supply chain. Therefore, the knowledge sharing has gradually attracted the attention of the academic community [1]. At present, foreign scholars pay more attention to practical research, which is mainly reflected in the construction of knowledge sharing system and demonstration of the importance of knowledge sharing through case studies. There are not many studies and mainly focus on knowledge sharing incentives, framework construction and realization methods. However, there are not many researches on the influencing factors of knowledge sharing among supply chain enterprises, and the corresponding empirical research is even more lacking. The Internet has realized a "super-efficiency market" for sharing usage rights from a technical perspective [2]. In the blockchain environment, shared objects have become blurred or completely disappeared. Knowledge is identified data information. In this sense, knowledge has no bystanders, only participants. The knowledge creation of supply chain enterprises in the blockchain environment is a bottom-up process, which means that knowledge creation is a decentralized process. Supply chain enterprises need an open decentralized environment for knowledge sharing.

Knowledge has been long recognized as a key resource for enterprises. The scope of knowledge sharing can range from binary information exchange among individuals to coordination of project teams to large-scale brainstorming activities for multinational companies. Knowledge sharing is a socialization process in which participants gain new knowledge and insights and transform them into new capabilities [3, 4]. This coincides with the concept of decentralization, because the shared environment is no longer limited, and can better reflect the openness and value of knowledge sharing. If supply chain companies want to make full use of knowledge at their destinations, the knowledge-led influence needs to expand, which embraces knowledge and promotes knowledge flow in the organization. However, knowledge is scattered in hundreds of separate subject databases, causing confusion and waste of knowledge sharing, especially the centralized network is full of "pseudo-knowledge", which requires the construction of a decentralized knowledge sharing mechanism. The decentralized mechanism that transcends the organization also faces problems such as trust and authenticity. The emergence of blockchain provides an effective way to solve these problems.

Blockchain is essentially a new method of data management, an open, transparent, and decentralized database. Blockchain is a technical system composed of distributed ledgers, point-to-point transmission, consensus mechanism, smart contracts, etc. This system can realize tamper-proof data storage, traceable data viewing, and trusted point-to-point transmission, which can solve the problem of trust construction under supervision [5]. The core meaning of this system is to realize the trustworthy circulation of value and promote the transformation from the "Internet of Information" to the "Internet of Value". It can be used as the basic protocol of the "Internet of Value" and its status is comparable to the current HTTP protocol. This article proposes the influencing factors of supply chain enterprise knowledge sharing, and then further studies it from an empirical perspective, and proposes targeted suggestions on this basis, in order to provide some substance for the knowledge sharing activities among supply chain enterprises.

\section{THEORETICAL FOUNDATION \\ 2.1 Decentralized Knowledge Sharing Mechanism}

On the consortium chain, the distributed knowledge after identification by the government, scientific research institutions, tourism enterprises and tourists, forms a decentralized knowledge graph library. Based on the distributed ledger, the knowledge storage is decentralized, and the consensus mechanism allows management to be decentralized. Reusing point-to-point transmission and smart contracts can solve the problems of trust, authenticity verification, and incentive distribution in the knowledge sharing process [6]. The existing knowledge storage subject writes knowledge to the alliance chain according to agreed rules, and the writing is carried out under a comprehensive feature information verification system to 
identify the authenticity of the knowledge. Knowledge users for business or research purposes send query requests to the alliance chain as needed. The alliance chain activates and executes the smart contract when receiving the query request, and the user will be calculated according to the pre-rule offer. After the consortium chain queries the user's needs, the query results are encapsulated as a knowledge set and passed to the user interface. Finally, the smart contract automatically deducts the queryer's fees according to the amount required for the query, and automatically completes the incentive distribution among the knowledge providers according to the contribution rate.

\subsection{Knowledge Sharing}

Knowledge sharing is a kind of communication behavior between the knowledge owner and the knowledge demander [7]. The knowledge owner transmits its knowledge through its own actions, words, or writing, and the demander perceives the expression of these behaviors. I recognize, understand, and digest this knowledge through my own imitation, listening, and reading.

Knowledge sharing is not only the transfer of knowledge from one party to the other, but also the willingness to help the other party understand the connotation of knowledge and learn from it, thereby transforming it into the other party's knowledge content, and ultimately developing the individual's ability to act. Knowledge sharing is not a static process, but a dynamic process. It is a continuous learning process, and it divides knowledge sharing into four stages: knowledge acquisition, communication, application, and absorption [8]. Knowledge sharing is regarded as the process of participating in the knowledge market of the enterprise's internal knowledge. Like other goods and services, the knowledge market also has buyers and sellers, and market participants can benefit from it. Scholars holding this view believe that knowledge sharing is similar to the market transaction process, and the benefits obtained by the two parties sharing knowledge in the sharing process are the driving force for their knowledge sharing.

\subsection{The Knowledge Chain Model among Supply Chain Enterprises}

From the perspective of supply chain knowledge sharing, whether knowledge sharing can be carried out, this involves the enterprise's intention and desire to participate in knowledge sharing. Enterprises must first have the intention and desire to carry out knowledge-sharing. Otherwise, knowledge sharing will not be possible. Secondly, if there are only sharing intentions and desires, but the enterprise lacks the ability to share knowledge such as absorption and transfer, then knowledge sharing cannot continue. Thirdly, if there is a sharing intention and desire, there is also a corresponding sharing ability, but without the process of sharing implementation, knowledge sharing cannot be carried out [9]. Finally, the characteristics of knowledge itself have a strong restriction on whether knowledge sharing can carry out effectively.

The knowledge can experience acquisition, selection, internalization, externalization, and innovation, and promote the economicalization of knowledge and the circular chain structure of organizational goals. According to the characteristics of the knowledge chain, a knowledge chain model among supply chain enterprises can be constructed, as shown in Fig. 1.

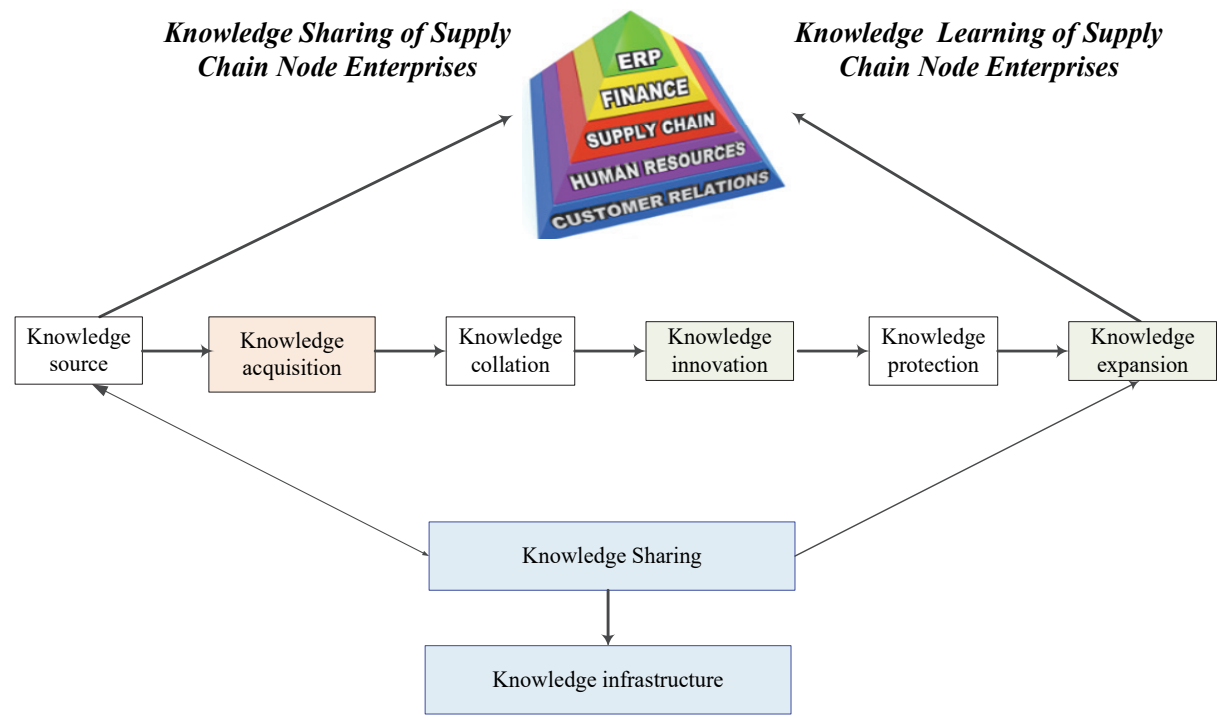

Figure 1 The knowledge chain among supply chain enterprises

\section{MODEL CONSTRUCTION AND HYPOTHESIS}

3.1 Supply Chain Enterprises on the Intention of Knowledge Sharing in the Blockchain Environment

Different enterprises of the knowledge subject in the blockchain environment transform the knowledge possessed by their members after learning into the knowledge of the organization, which improves the organization's activities and further spread, and stores the process within the organization [10-13]. Enterprise organizational learning is based on the group that a person learns. At present, supply chain companies have not yet formed a unified standard for the composition of organizational learning capabilities. The organizational learning ability of supply chain enterprises is the knowledge application ability, which effectively 
decomposes the organizational learning ability into seven aspects: discovery ability, invention ability, selection ability, execution ability, promotion ability, anticonduction ability, and knowledge management ability. Based on the above analysis, three indicators are used: the nature of the company, the number of years the company has established, and the scale of the company to measure the company's knowledge sharing intentions.

Hypothesis 1: Knowledge plays a role in promoting the influence of knowledge sharing intention.

Hypothesis 2: Blockchain environment plays a role in promoting the influence of knowledge sharing intention.

Hypothesis 3: Blockchain technology has played a role in promoting the influence of knowledge sharing intention.

The intention of knowledge sharing is to express the knowledge sharing relationship between enterprises. The intent of knowledge sharing includes both transaction costs and information exchange costs among companies. Companies will use the least interactive cost to achieve their goals. In the case of a certain blockchain technology, the higher the intention of knowledge sharing among enterprises, the lower the cost of information exchange between enterprises, so that mutual knowledge sharing activities are active, which can ensure the smooth progress and supply of knowledge sharing activities, harmony and endurance of chain cooperation. Hence, we can obtain the following relationship.

Hypothesis 4: In the blockchain environment, the knowledge sharing intention plays an intermediary role in the influence of knowledge sharing behavior.

Since the cooperation among supply chain enterprises is based on the division of functions, each node enterprise only produces its own competitive products or services. In this way, the stickiness of the technical knowledge of each member enterprise is reduced in the process of knowledge sharing among supply chain enterprises. This is particularly prominent, and this effect is called the barrier property of technical knowledge.

Hypothesis 5: Blockchain technology has played a role in promoting the influence of knowledge sharing behavior.

\subsection{Knowledge sharing intention on Knowledge Sharing Behavior in the Blockchain Environment}

In the blockchain environment, each member company of the supply chain system has differences in storage methods, transmission forms and knowledge means. The foundation of collaboration enables all supply chain members to share and acquire the knowledge they need. In the supply chain, each member company hopes to gain a strong position in the distribution of benefits through the knowledge that it owns but other companies cannot grasp. To a certain extent, sharing knowledge is contrary to corporate behavior. During this period, the main body of knowledge in supply chain enterprises should actively guide the knowledge sharing intentions of various enterprises and play a positive role. Each company member hopes to gain its own status in the distribution of benefits through knowledge that it has mastered but other companies cannot.

Hypothesis 6: The knowledge subject regulates the relationship between knowledge and knowledge sharing intention.

The information technology of the supply chain system is the external material guarantee for the establishment and perfection of the knowledge sharing platform among supply chain enterprises. The establishment of a complete knowledge sharing technology platform also requires strong compatibility of the information system of each enterprise, and the establishment of a sharing platform requires the active participation of member companies. The blockchain environment constructed by blockchain technology provides a guarantee for the knowledge sharing of supply chain enterprises to a certain extent. There is no effective knowledge sharing incentive mechanism in the blockchain environment, and to a certain extent, it will inhibit the willingness of enterprises to share knowledge. In a wellestablished blockchain environment, the corporate culture adopted by upstream and downstream companies in the supply chain has the characteristics of consistency and openness, and the degree of trust among member companies. In the existing blockchain environment, it is difficult for knowledge subjects to adjust the blockchain environment and knowledge sharing intentions. The stickiness of the technical knowledge of each member company is particularly prominent in the process of knowledge sharing between supply chain companies, and it is difficult for the knowledge subject to adjust the existing blockchain technology and knowledge sharing intentions.

Hypothesis 7: The knowledge subject regulates the relationship between the blockchain environment and the knowledge sharing intention.

Hypothesis 8: The knowledge subject regulates the relationship between blockchain technology and knowledge sharing intentions.

\subsection{The Knowledge Sharing among Supply Chain Enterprises in the Blockchain Environment}

According to the supply chain of enterprise knowledge sharing, it based on the blockchain environment can have constructed in Fig. 2. The supply chain knowledge base collects knowledge from various node enterprises outside the supply chain, and forms the knowledge that meets the requirements of the node enterprises through processing and sorting. While each node enterprise provides knowledge to the knowledge base, it also obtains knowledge from the knowledge base and other node enterprises. In the model, the inner circle is the knowledge storage layer; the outer circle is the knowledge-sharing layer. Each node enterprise submits knowledge demand signals to the knowledge storage layer, thus forming a connected knowledge chain.

Based on this analysis, we divide the supply chain knowledge sharing influencing factor system under the blockchain environment into the following three subsystem dimensions, namely the knowledge factor dimension, the blockchain environment dimension, and the blockchain technology dimension. Each dimension will construct the corresponding influencing factor sub-models. The three-dimensional subsystems together constitute the overall research framework system of the influence factors. 


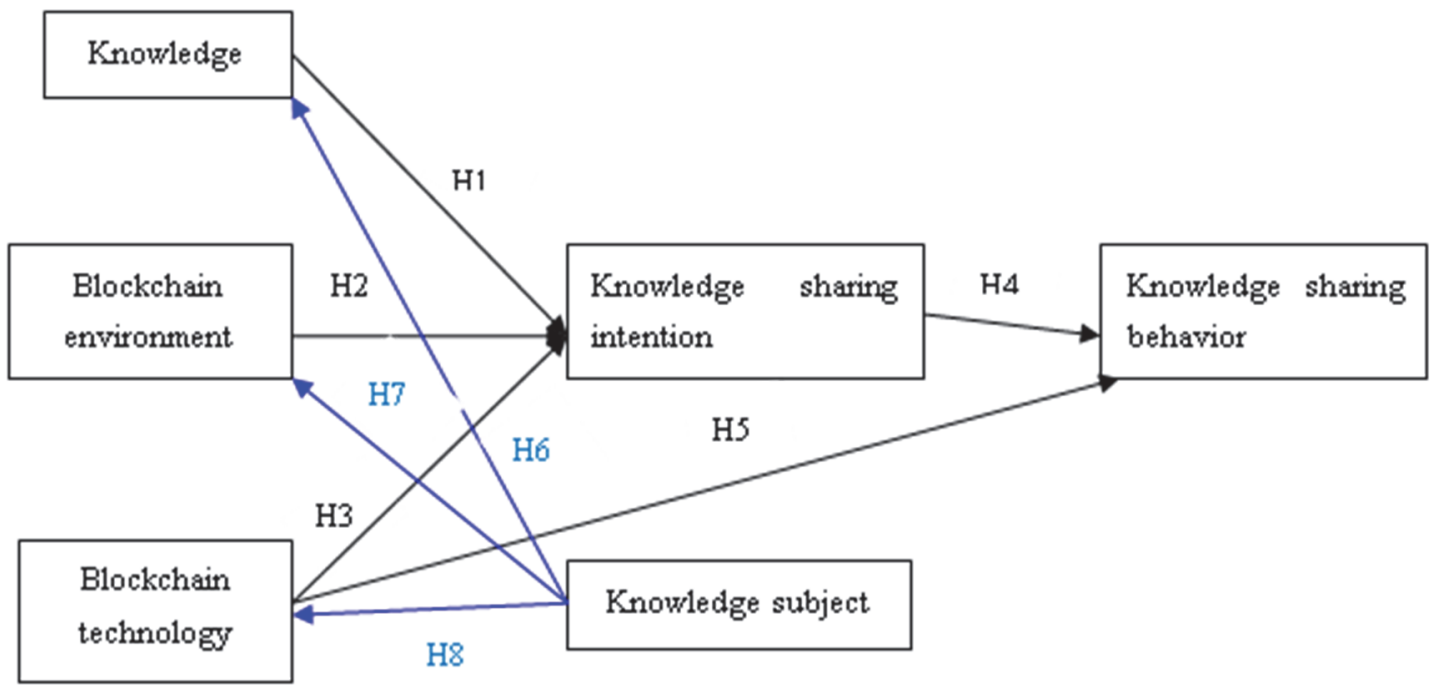

Figure 2 The construct of supply chain knowledge

1) Knowledge factor dimension

Whether an enterprise has the intention and willingness to share knowledge is a prerequisite for the supply chain to carry out knowledge sharing activities. If the enterprise does not have this willingness, the knowledge sharing will not be possible [14]. Therefore, the influencing factor dimension of intention and desire is an important subsystem of supply chain knowledge sharing. The willingness of supply chain knowledge sharing is affected by the trust among enterprises, the level of cooperation among enterprises, and the incentive mechanism for sharing. These factors positively or negatively affect the willingness level of enterprises.

2) Blockchain environment dimension

As an enterprise, only the willingness to share knowledge cannot complete the knowledge sharing activities, and it needs the ability to participate in knowledge sharing. The blockchain environment determines which knowledge to share with supply chain companies is far from enough. It also requires the ability to transfer knowledge. The so-called transfer capability here does not mean simply passing knowledge to supply chain companies. Similarly, as a knowledge receiver, it is not enough to just take the knowledge of the supply chain enterprise, but also to learn, digest, absorb, and use, integrate new knowledge with its own knowledge base, and improve the enterprise through the application of new knowledge.

3) Blockchain technology dimension

Blockchain technology is the specific implementation stage of knowledge sharing activities. The process of sharing implementation is the process of mutual communication between the two parties [15]. Communication plays an important role in the implementation stage of knowledge sharing. The form of communication may be face-to-face, or through telephone, internet, video or other forms. The frequency of communication also depends on the actual situation. Both parties of knowledge sharing will be affected by many factors in the communication process, such as the influence of corporate culture and corporate values. Different cultures and values can easily lead to conflicts between the two parties, which will adversely affect communication. In addition, the construction of information platforms between enterprises also has an impact on the communication process. An effective information platform can greatly shorten the distance between enterprises and improve the efficiency of communication. At the same time, the information platform construction of the blockchain technology is also conducive to the transfer of shared knowledge among enterprises, thereby promoting the supply chain knowledge sharing activities.

\section{QUESTIONNAIRE DESIGN AND EMPIRICAL METHODS 4.1 Questionnaire Design and Distribution}

The data for this study were mainly obtained through questionnaire surveys. The subjects of the survey involved key employees of the supply chain partner companies in the manufacturing industries of electronics and electrical appliances, and so on. Due to the concentration of manufacturers in these industries, the high level of substitution of different brand products, and fierce market competition enhance the competitiveness of enterprises; most of the industry hopes to grasp the development trend of upstream and downstream enterprises through knowledge sharing, and obtain knowledge that matches the core enterprises to establish a stable supply [16]. The main body of the survey questionnaire contains three parts: basic corporate information, a scale of factors affecting knowledge sharing, and a scale of knowledge sharing effects among supply chain companies. This study uses the 5-point scale to design influencing factors and knowledge sharing effect scales, ranging from 1 to 5 to indicate very disagree, disagree, unsure, agree and very agree. In order to ensure the quality of the questionnaire design, a small sample test was first conducted in Chongqing City, and the questions of the questionnaire were adjusted and corrected based on the feedback results.

The official questionnaires were distributed in Chongqing, Chengdu, Guiyang, Beijing and other cities by door-to-door distribution and mailing. 200 questionnaires were issued, and 180 questionnaires were recovered, of which 165 were valid questionnaires, and the recovery rate was 83 . The effective recovery rate is 82.3. Among them, 55 were state-owned and state-controlled enterprises $(27.5 \%), 23$ were collective cooperative enterprises $(11.5 \%), 43$ were private/private enterprises $(21.5 \%)$, and 
60 were joint ventures $(30 \%), 19$ foreign-invested enterprises $(9.5 \%)$. As far as the interviewees are concerned, 86 are those over 30 years old. 79 employees have worked for more than 5 years and accounted for $68 \%$. Those with a college degree account for $92 \%$. Regardless of work experience, experience, or academic qualifications, they should have a relatively good familiarity and sensitivity to the questions covered by the questionnaire. Therefore, this study adopts SPSS software carried on the structural formula modeling. Other data statistics analysis is completed by the statistical software.

\subsection{Model Design}

When the latent variable is a continuous variable and the moderating variable is a categorical variable, group regression can be implemented to test the moderating effect of moderating variables on a specific path relationship, and then the regression coefficient differences of different groups can be compared. If the difference is significant, the moderating effect is significant. In this paper, the latent variable knowledge subject is the continuous variable after factor analysis, the knowledge sharing intention is the observed variable, the adjustment variable blockchain environment, the attribute of the knowledge itself and the blockchain technology are the categorical variables. In view of this, this article adopts group regression analysis, examines the regulatory effect of the knowledge subject in the blockchain environment, the attributes of knowledge itself, and blockchain technology as a moderating variable in the relationship between knowledge sharing awareness.

When doing moderating effect analysis, the latent variable and moderating variable are usually transformed centrally (that is, variable minus its mean value). This article mainly chooses commonly used adjustment models, that is, assumes that Observed variable $Y$ and latent variable $X$ have the following relationship.

$Y=a X+b M+e$

For a fixed constant $M$, this is the linear regression of $Y$ to $X$. The relationship between $Y$ and $X$ is described by the regression coefficient a, which is a linear function of $M$, and $b$ measures the size of the adjustment effect.

In the moderating effect analysis, the status of knowledge, blockchain technology and blockchain environment can be symmetrical, and any of them can be interpreted as a moderating variable. The moderating effect between latent variables is clear. For example, in the moderating effect, which is the independent variable and which is the moderating variable is very clear, and the two cannot be interchanged in a certain model. Knowledge, blockchain technology and blockchain environment have the same role in regulating the status of knowledge sharing intentions.

\subsection{Analysis of Reliability and Validity}

The credibility of this questionnaire is tested using the Cronbach coefficient. The moderating effect analysis method depends on the measurement level of the independent variable and the moderating variable. Variables can be divided into two categories, one is category variables, including fixed and ordinal variables, and the other is continuous variables, including fixed distance and fixed ratio variables. When the value of ordinal variable is relatively large and the interval is relatively uniform, it can also be approximated as a continuous variable. Tab. 1 lists the methods for analyzing the moderating effects of significant variables. The analysis results of the SPSS statistical software show that the Cronbach a coefficient of each variable is greater than 0.6. The total Cronbach a coefficient of the question paper is 0.696 (see Tab. 1), indicating that the corresponding variables of each factor have a strong correlation, and the internal consistency is better.

For the validity test, this article adopts the factor analysis method to investigate the factor load of each factor to the corresponding variable. In social sciences, the absolute value of the factor load greater than 0.4 is considered effective. The factor loads of all observed variables in this questionnaire are greater than 0.6 (See Tab. 1). Therefore, each factor has a strong explanatory power for the corresponding latent variables, the quality of the questionnaire is relatively high, and further research can be carried out.

\begin{tabular}{|c|c|c|c|}
\hline Latent variable $(X)$ & Observed variable $(Y)$ & Cronbach & Factor loading \\
\hline $\begin{array}{c}\text { Business organization } \\
\text { Learning ability }\end{array}$ & $\begin{array}{l}\text { Knowledge absorptive capacity } \\
\text { Knowledge integration ability } \\
\text { Knowledge application ability }\end{array}$ & 0.73 & $\begin{array}{l}0.82 \\
0.83 \\
0.79\end{array}$ \\
\hline Technical knowledge & $\begin{array}{l}\text { Corporate specificity } \\
\text { Modularity level }\end{array}$ & 0.74 & $\begin{array}{l}0.80 \\
0.78\end{array}$ \\
\hline $\begin{array}{l}\text { The Perfection level of knowledge } \\
\text { sharing platform }\end{array}$ & $\begin{array}{c}\text { Enterprise information level } \\
\text { Information system participation enthusiasm }\end{array}$ & 0.66 & $\begin{array}{l}0.71 \\
0.74\end{array}$ \\
\hline $\begin{array}{l}\text { Perfection level of the incentive } \\
\text { system }\end{array}$ & $\begin{array}{l}\text { Knowledge sharing evaluation, } \\
\text { Level of business support }\end{array}$ & 0.68 & $\begin{array}{l}0.69 \\
0.84\end{array}$ \\
\hline Inter-firm compatibility level & $\begin{array}{l}\text { Corporate culture consistency and openness, and } \\
\text { cultural compatibility }\end{array}$ & 0.70 & $\begin{array}{l}0.53 \\
0.68\end{array}$ \\
\hline
\end{tabular}

\subsection{Research Model Inspection}

After completing the reliability and validity test of the questionnaire, the $p$-value, $\mathrm{C}-\min / d f$, and other indicators are used to measure the fitting effect of the research model, and the relevant coefficients are shown in Tab. 2. For the entire model, except for the $R E$ of 0.06 , which is slightly higher than the better model fitting value of 0.05 , the rest of the model fitting values meet the requirements of a better fit. Statistics lower than 0.1 indicates an ideal fit, and lower than 0.05 indicates a better fit. This shows that the structural equation model established in this study has a better fitting effect. 
Table 2 Model coupling index

\begin{tabular}{|c|c|c|}
\hline Fitting index & Fitting index & $\begin{array}{c}\text { The model } \\
\text { fitting well }\end{array}$ \\
\hline Statistics & 0.06 & $>0.05$ \\
\hline C - min/df & 0.52 & $<2.00$ \\
\hline Goodness of fit index & 0.94 & $>0.90$ \\
\hline Adjust the goodness of fit index & 0.93 & $>0.90$ \\
\hline Approximate root mean square error & 0.06 & $>0.05$ \\
\hline Relative fit index & 0.99 & $>0.95$ \\
\hline Standard Fit Index & 0.98 & $>0.95$ \\
\hline TL index & 0.99 & $>0.95$ \\
\hline Increasing fit index & 0.99 & $>0.95$ \\
\hline
\end{tabular}

\subsection{Research Hypothesis Test}

According to the structural equation model analysis, the proposed hypothesis is analyzed, and the following results are obtained:

Fig. 3 is the structural equation model measurement. Tab. 3 shows the standardized path coefficient between latent variable and their corresponding $p$-value. Among them, the $p$-value is used to test the significance of the relationship among variables. It is generally considered that $p<0.05$ coefficient is significant, and $p<0.01$ coefficient is very significant.

Through the above empirical analysis, from hypothesis 1 to hypothesis 8 , they have verified. Assume that the $p$ values of hypothesis 2 , hypothesis 4 , hypothesis 6 , and hypothesis 7 are not significant and fail the test. Enterprise organizational learning ability and the attributes of technical knowledge barriers have a significant impact on knowledge acquisition, but have no significant impact on the intention of knowledge sharing, mainly through knowledge acquisition to affect the cooperation behavior. Knowledge sharing platform, incentive mechanism and inter-firm compatibility level all have a significant impact on knowledge acquisition and cooperation behavior.

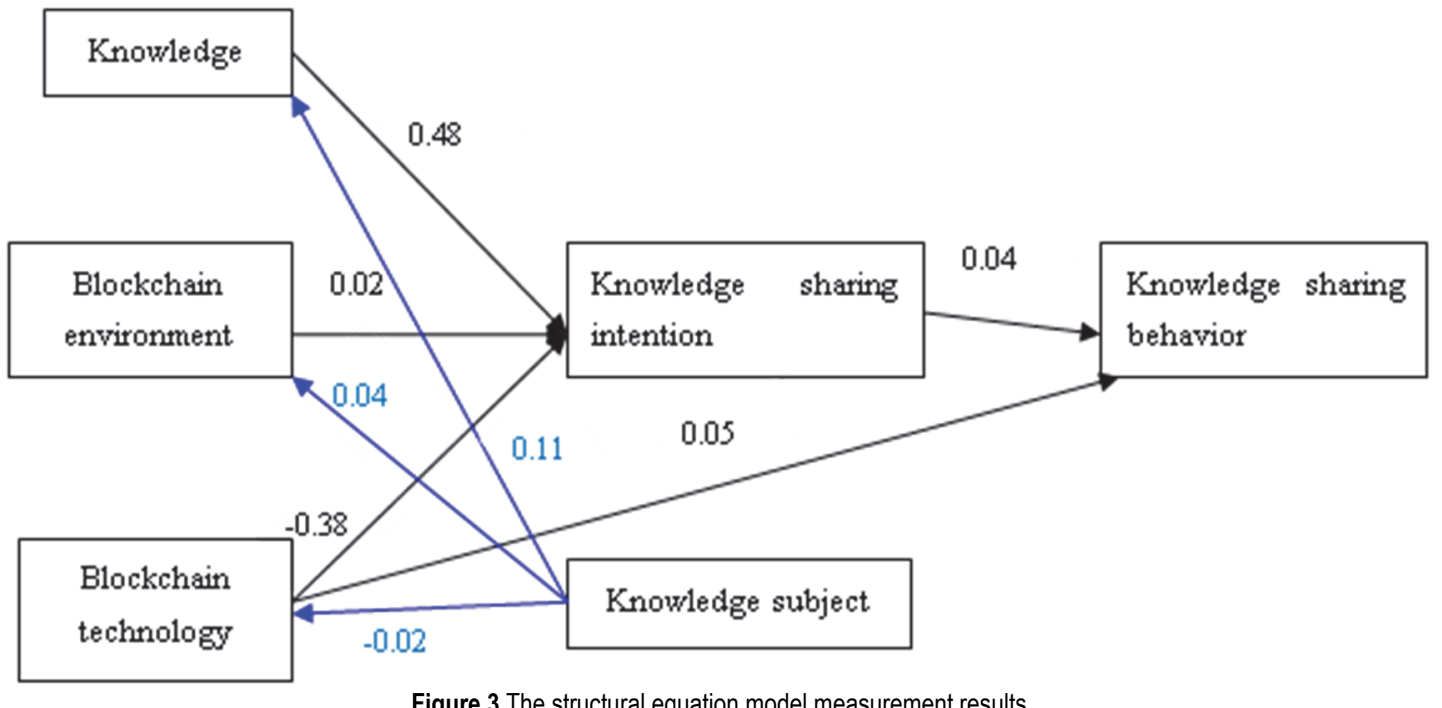

Figure 3 The structural equation model measurement results

Table 3 Standardized path coefficient

\begin{tabular}{|c|c|c|c|c|}
\hline Hypothesis & Path direction & Path coefficient & $p$-value & Result \\
\hline Hypothesis 1 & $\begin{array}{l}\text { The enterprise nature plays a moderating role in the influence of the } \\
\text { blockchain environment on the knowledge sharing intention }\end{array}$ & 0.48 & 0.01 & Passed \\
\hline Hypothesis 2 & $\begin{array}{c}\text { Blockchain environment plays a role in promoting the influence of } \\
\text { knowledge sharing intention }\end{array}$ & 0.02 & 0.06 & Not Passed \\
\hline Hypothesis 3 & $\begin{array}{c}\text { Blockchain technology has played a role in promoting the influence of } \\
\text { knowledge sharing intention }\end{array}$ & -0.38 & 0.02 & Not Passed \\
\hline Hypothesis 4 & $\begin{array}{l}\text { In the blockchain environment, the knowledge sharing intention plays an } \\
\text { intermediary role in the influence of knowledge sharing behavior }\end{array}$ & 0.11 & 0.08 & Passed \\
\hline Hypothesis 5 & $\begin{array}{l}\text { Blockchain technology has played a role in promoting the influence of } \\
\text { knowledge sharing behavior }\end{array}$ & 0.05 & 0.01 & Passed \\
\hline Hypothesis 6 & $\begin{array}{l}\text { The knowledge subject regulates the relationship between knowledge and } \\
\text { knowledge sharing intention }\end{array}$ & 0.11 & 0.06 & Passed \\
\hline Hypothesis 7 & $\begin{array}{c}\text { The knowledge subject regulates the relationship between the blockchain } \\
\text { environment and the knowledge sharing intention }\end{array}$ & 0.04 & 0.07 & Not passed \\
\hline Hypothesis 8 & $\begin{array}{l}\text { The knowledge subject regulates the relationship between blockchain } \\
\text { technology and knowledge sharing intentions }\end{array}$ & -0.02 & 0.03 & Not passed \\
\hline
\end{tabular}

\section{CONCLUSIONS}

As far as the current situation in our country is concerned, the problems of asymmetric information and lack of trust among supply chain enterprises are the biggest obstacles to the development of logistics. This article introduces the three main problems in the supply chain system and the principle of blockchain, the blockchain technology is applied to the supply chain system to solve the enterprises of the supply chain system. However, it is not easy to implement blockchain. This is not just a technical problem. The biggest problem is how to make companies in the supply chain trust a decentralized platform. This requires maximum Information resource sharing and collaboration. Just like the development process of the Internet economy in China, the blockchain may also experience many setbacks and distrust, which requires scientific researchers and social-related 
enterprises to work together to develop the potential of the blockchain. It is believed that the era of blockchain will usher in the next few years, which will be a huge impact on trust issues.

\section{REFERENCES}

[1] Zhang, H., Li, S., Yan, W., Jiang, Z., \& Wei, W. (2019). A Knowledge Sharing Framework for Green Supply Chain Management Based on Blockchain and Edge Computing. Smart Innovation, Systems and Technologies, 155. https://doi.org/10.1007/978-981-13-9271-9_34

[2] Bo, Y. \& Meifang, Y. (2020). Construction of the knowledge service model of a port supply chain enterprise in a big data environment. Neural Computing and Applications. https://doi.org/10.1088/1742-6596/1550/3/032170

[3] Li, S., Zhang, H., Yan, W. et al. (2020). A hybrid method of blockchain and case-based reasoning for remanufacturing process planning. Journal of Intelligent Manufacturing. https://doi.org/10.1007/s10845-020-01618-6

[4] Ghode, D., Yadav, V., Jain, R., \& Soni, G. (2020). Adoption of blockchain in supply chain: an analysis of influencing factors. Journal of Enterprise Information Management, 33(3), 437-456. https://doi.org/10.1108/JEIM-07-2019-0186

[5] Wang, J. \& Liu, L. (2019). Study on the mechanism of customers' participation in knowledge sharing. Expert Systems. https://doi.org/10.1111/exsy.12367

[6] Abbas, Y., Martinetti, A., Moerman, J. J., Hamberg, T., \& Dongen, L. A. M. V. (2020). Do you have confidence in how your rolling stock has been maintained? ablockchain-led knowledge-sharing platform for building trust between stakeholders. International Journal of Information Management, 55, 10. https://doi.org/10.1016/j.jijinfomgt.2020.102228

[7] Lin, X., Li, J., Wu, J., Liang, H., \& Yang, W. (2019). Making knowledge tradable in edge-ai enabled IoT: a consortium blockchain-based efficient and incentive approach. IEEE Transactions on Industrial Informatics, 15(99), 6367-6378. https://doi.org/10.1109/TII.2019.2917307

[8] Li, Z., Liu, X., Wang, W. M., Vatankhah Barenji, A., \& Huang, G. Q. (2018). Ckshare: secured cloud-based knowledge-sharing blockchain for injection mold redesign. Enterprise Information Systems, 1-33. https://doi.org/10.1080/17517575.2018.1539774

[9] Li, J., Greenwood, D., \& Kassem, M. (2019). Blockchain in the built environment and construction industry: a systematic review, conceptual models and practical use cases. Automation in Construction, 102(JUN.), 288-307. https://doi.org/10.1016/j.autcon.2019.02.005

[10] Wanlu, Z. (2019). Research on the influencing factors of enterprise business model innovation in internet platform: a case study based on didi. Value Engineering, 038(006), 3841.

[11] Radej, B., Drnovsek, J., \& Beges, G. (2017). An overview and evaluation of quality-improvement methods from the manufacturing and supply-chain perspective. Advances in Production Engineering \& Management, 12(4), 388-400. https://doi.org/10.14743/apem2017.4.266

[12] Jiang H. \& Liu C. Y. (2019). Scheduling Optimization of Cloud Resource Supply Chain through Multi-Objective Particle Swarm Optimization. International Journal of Simulation Modelling, 18(1), 163-174. https://doi.org/10.2507/IJSIMM18(1)CO3

[13] Zic, J. \& Zic, S. (2020). Multi-criteria decision making in supply chain management based on inventory levels, environmental impact and costs. Advances in Production Engineering \& Management, 15(2), 151-163. https://doi.org/10.14743/apem2020.2.355

[14] Guiyan, Z., Xiaomeng, M., \& Benfa, L. (2018). Research on the mechanism of knowledge and intelligence sharing in enterprises under big data: from the perspective of status: triving motivation and opinion dynamics. Expert Systems, 36, e12313- e12316. https://doi.org/10.1111/exsy.12313

[15] Liu, S., Hu, Y., Zhang, X., Li, Y., \& Liu, L. (2020). Blockchain service provider selection based on an integrated bwm-entropy-topsis method under an intuitionistic fuzzy environment. IEEE Access, PP(99), 1-1. https://doi.org/10.1109/ACCESS.2020.2999367

[16] Li, G., Dong, M., Yang, L. T., Ota, K., \& Li, J. (2019). Preserving edge knowledge sharing among iot services: a blockchain-based approach. IEEE Transactions on Emerging Topics in Computational Intelligence, PP(99), 1-14.

\section{Contact information:}

Yi CHAl

School of Management, Jilin University, China

Quanxi LI

(Corresponding author)

School of Management, Jilin University,

No. 2699 Qianjin Street, Changchun City, 130012, China

E-mail: liquanxi_jlu@163.com 